Article

\title{
Targeting the Intestine to Prevent Glucocorticoid Induced Osteoporosis
}

\author{
Laura R. McCabe * \\ Department of Physiology, Biomedical Imaging Research Center, Michigan State University, Biomedical \\ Physical Science Building, 567 Wilson Road, East Lansing, MI, 48824, USA \\ * Correspondence: mccabel@msu.edu
}

Published: 30 October 2020

\begin{abstract}
Gut-bone signaling is recognized as contributing to bone health. The gut has two key compartments: 1) the microbiota; which contains more than a trillion bacteria and 2) the intestinal barrier which prevents microbiota and their factors leaking into the lamina propria and blood stream. Chemically inducing barrier leaks causes osteoporosis. Similarly; conditions of osteoporosis (e.g., menopause; diabetes; hyperparathyroidism) are associated with intestinal microbiota dysbiosis and barrier leaks. Glucocorticoid induced osteoporosis (GIO) is also associated with microbial dysbiosis. By depleting the microbiota with antibiotics and performing fecal transplants; we demonstrate a key role for the microbiota in mediating GIO. Factors such as probiotic Lactobacillus reuteri (LR) modify the intestinal microbiota and prevent GIO. LR also prevented glucocorticoid induced intestinal barrier leaks. Treating mice with an intestinal barrier enhancer demonstrats an important role for barrier integrity in preventing GIO. Similarly decreases in osteoblast number; increased marrow adiposity; and increased osteoblast/osteocyte death induced by glucocoroticoid treatment were prevented by LR; the barrier enhancer; and by microbiota depletion. Preventing suppression of Wnt10b is suggested to prevent GIO. Our data support a role for changes in both the microbiota and intestinal barrier function in GIO; making these two components of the gut important therapeutic targets
\end{abstract}

Publisher's Note: MDPI stays neutral with regard to jurisdictional claims in published maps and institutional affiliations.

(C) 2020 by the authors. Submitted for possible open access publication under the terms and conditions of the Creative Commons Attribution (CC BY) license (http://creativecommons.org/licenses/by/4.0/). 